\title{
Emergency preparedness for long lasting releases - overview and conclusions
}

\author{
F. Gering ${ }^{1}$, B. Gerich ${ }^{1}$, K. Arnold ${ }^{1}$, T. Peltonen ${ }^{2}$, T. Duranova ${ }^{3}$, A. Bujan ${ }^{3}$, J. Duran $^{3}$, \\ L. Bohun ${ }^{3}$, M. Montero ${ }^{4}$, C. Trueba ${ }^{4}$, L. Puijker ${ }^{5}$, C. Twenhöfel ${ }^{6}$ and H. de Vries ${ }^{7}$ \\ ${ }^{1}$ BfS - Federal Office for Radiation Protection, Ingolstaedter Landstr. 1, D-85764 Oberschleissheim, Germany. \\ ${ }^{2}$ Radiation and Nuclear Safety Authority (STUK), Finland, Säteilyturvakeskus, Finland. \\ 3 VUJE, Inc., Okružná 5, 91864 Trnava, Slovakia. \\ ${ }^{4}$ CIEMAT, Environment Department, Av. Complutense 40, 28040 Madrid, Spain. \\ ${ }^{5}$ KWR Watercycle Research Institute, Groningenhaven 7, 3430 BB Nieuwegein, The Netherlands. \\ ${ }^{6}$ RIVM, Antonie van Leeuwenhoeklaan 9, 3721 MA Bilthoven, The Netherlands. \\ ${ }^{7}$ KNMI Royal Netherlands Meteorological Institute, 3730 AE De Bilt, The Netherlands.
}

\begin{abstract}
The Fukushima accident has demonstrated the possibility of long-lasting releases of radionuclides from a nuclear power plant over several weeks. Within the framework of the "PREPARE" research project one work package focused on testing emergency preparedness arrangements in various countries for the special case of long lasting emissions. The aim of this study was to test whether protection strategies included in current nuclear emergency planning in various European countries could adequately reduce the radiological consequences of Nuclear Power Plant accidents with long lasting severe releases. This paper presents an overview and the conclusions of this study, more details of the work can be found in the following articles.
\end{abstract}

Keywords: radiological consequence assessment / severe accident scenario / long lasting release

\section{Introduction}

In all countries with nuclear installations detailed emergency management strategies have been developed in the past. In nearly all cases such strategies are based on accident scenarios where the duration of the release of radionuclides to the environment is limited to some hours or at maximum a few days. The Fukushima accident has demonstrated the possibility of long-lasting releases of radionuclides from a nuclear power plant over several weeks. This made it necessary to test the current off-site nuclear emergency planning in European countries with accident scenarios based on lessons learned from the Fukushima accident and to derive recommendations how to improve the current planning. The tests should demonstrate whether protective measures foreseen in the current emergency planning could adequately reduce the radiological consequences of Nuclear Power Plant (NPP) accidents with long lasting severe releases similar to the release from the FukushimaDaiichi NPP.

The objectives of the study were two-fold: first, to evaluate - using realistic accident scenarios - the radiological consequences of these accidents according to the type of reactor involved and the varying conditions that influence the pattern of dispersion and deposition of radioactive contamination; and second, to explore whether these effects can be reduced and properly managed with the current emergency management strategies and procedures.

\section{Methods}

The first step of the study was to define hypothetical source terms leading to long-lasting releases of radionuclides which are applicable to nuclear installations in various European countries. In total 9 source terms have been identified for different reactor types such as Pressurised Water Reactor (PWR), Boiling Water Reactor (BWR) and Water-Water Energy Reactor (VVER). The accident scenarios leading to the accidental releases of radioactivity included core melt followed by total core damage scenarios in all cases, combined with containment damage in 7 cases, while in the remaining 3 cases releases occurred through venting. The duration of the releases varied from 22 to $188 \mathrm{~h}$. Most of the scenarios can be grouped in the INES 7 scale (with 2 cases rated as INES 6 and one case as INES 5) and the amount of ${ }^{131}$ I released in the 10 scenarios varied between 3 and $600 \mathrm{PBq}$. For more details about the source terms see (Bujan et al., 2016).

In the second step, appropriate weather data for the release scenarios were identified. As four countries participated in the work activity, four different weather data sets were used. All in all seven nuclear power plant sites in four European countries were included in the simulations. Some countries selected numerical weather data covering a complete year, typical for the average weather conditions in the country, whereas others used statistically derived typical weather conditions for particular times. In one case also weather conditions monitored at the site 
with a 10 min interval was used. For each of the 10 source terms between 46 and 365 weather scenarios were considered. The combination of weather and source term resulted in several thousands of dispersion calculations. This large set of calculations resulted in a wide range of results, which provided an excellent basis for the final evaluation. For more details about the accident scenarios see (Montero et al., 2016).

The results of the calculations were evaluated in terms of dose and concentration fields. In particular areas and distances where national intervention criteria were exceeded have been identified. For more details about the assessment of radiological consequences see (Peltonen et al., 2016).

\section{Conclusions}

In the final step, the results were compared against the existing national emergency management planning procedures. The evaluation concentrated on the question whether existing emergency preparedness and response capabilities in Europe are appropriate for nuclear accidents with long lasting releases. Several key questions were raised and a summary of the conclusions and answers for those questions are presented in the following:

- Are the current emergency planning zones sufficiently reflecting the potential radiological impact of long-lasting releases?

In most release scenarios the areas calculated for protective actions did not exceed current planning zones. In the cases where these distances were exceeded, the amount of affected population remained quite small. Only for Germany the old (i.e. before 2014) planning zones were exceeded in several cases, but the new and enlarged planning zones (introduced in 2014) proved to be large enough to cover areas with protective actions in most cases. While affected areas are typically getting larger with increasing release duration, the maximum distances for protective actions are decreasing.

- Is the current concept of emergency planning zones as being implemented in various countries reasonably applicable for long lasting releases?

The number of sectors affected increased clearly with the duration of the release. If the release duration was less than $12 \mathrm{~h}$, the affected sector was limited to less than $90^{\circ}$ in most cases. For very long lasting releases, however, the whole circle area (around the release point) could be affected (up to $360^{\circ}$ ). The results from one country showed, that in most cases not more than 3 sectors $\left(\leq 90^{\circ}\right)$ were affected for evacuation and sheltering, but more for partial sheltering and ITB for children. It can be concluded that planning for implementation of protective actions within $90^{\circ}$-segments is only appropriate for releases shorter than $12 \mathrm{~h}$. For those protective actions extending to larger distances from the release site (Iodine thyroid blocking, partial sheltering), more sectors may be affected than for short range actions like evacuation.

- Are current intervention criteria appropriate for long lasting releases?

- Related to the discussion about intervention criteria, the following conclusions were derived, differing in the various countries.
- According to this assessment the intervention criteria (in Nordic countries) seem to be appropriate for these kinds of long-lasting releases. As the considered scenarios were severe INES level 7 accidents (for Finland), it can be assumed that the criteria are suitable for less severe scenarios also.

- In the Nordic countries "partial sheltering" is included as an additional protective action in emergency planning and response, which does not exist in most other countries. It will primarily be implemented at longer distances from the accident site or to protect children and pregnant women specifically. The intervention criteria for partial sheltering are lower than that for normal sheltering and thus the amount of population affected by this countermeasure can become rather large. During partial sheltering it is recommended not to spend time outdoors unnecessarily. Such a protective action is much easier to recommend than totally sheltering indoors.

- There may be problems in the implementation of protective measures in the short term, since the release time can exceed even the stipulated time to determine the need for evacuation or temporary relocation.

- In case of long lasting severe releases the critical situation can occur, that intervention criteria for protective actions are not exceeded in any 7-day-interval (for which some of the reference levels are currently defined in Germany), but the total dose over the release period by far exceeds the reference level. This problem can become even more serious, if intervention criteria are based on an integration period of 2 days. Thus it might be concluded that the integration period for doses, which are compared against intervention criteria, should cover the release duration for long-lasting releases.

- Is the ICRP reference level for residual dose (over 1 year) exceeded?

The current intervention criteria in all countries assured, that the residual dose in the first year (ICRP reference level) did not exceed $100 \mathrm{mSv}$. Even if the general findings support the current planning, some shortcomings were identified:

- For very long lasting releases (i.e. more than 24-48 h) sheltering indoors inside the EPZ is probably not as suitable as in cases of a short release.

- In case of long lasting severe releases the protective action "sheltering" may impose additional problems (e.g. the danger of being forced to order late evacuation even during passage of the plume, lifting of sheltering in one area while ordering in an another area), which endangers the applicability of the action in general.

- In case of long lasting severe releases a one-time intake of stable iodine often is not sufficient for protecting the population against large thyroid doses. Multiple intakes of stable iodine tablets may not be sufficiently considered in emergency planning.

- What is the potential impact on drinking water?

Besides the terrestrial pathways, one country, the Netherlands, performed also assessment for drinking water. Some of the conclusions were:

- In case of a nuclear accident surface water can be contaminated by high levels of radionuclides and is not suitable for drinking water production. 
- Advanced treatment processes as ion-exchange and reversed osmosis do remove radionuclides effectively, but these are not common practice.

- Soil passage (dune infiltration, river bank filtration, groundwater) are a safe barrier for I-131 and Cs-137.

- If surface water is the main direct source for drinking water production, emergency plans for drinking water supply are needed.

- Drinking water utilities in the European countries are required by the EU Drinking Water Directive to provide emergency drinking water in case of a major accident, including nuclear accidents.

Due to the wide variability of source terms and weather conditions, this study provided a very good basis to evaluate the existing emergency preparedness and response capabilities in Europe with respect to long lasting releases. The evaluation was performed with the input from Germany, Finland, Slovak Republic and Spain. In general one can conclude that the current EP\&R of these four countries, as representatives of European regulations, are fit for purpose-nevertheless some areas for further improvement could be identified.

During the testing of the current off-site nuclear emergency planning in European countries with accident scenario simulations as described above, some issues could not be analysed or investigated in sufficient detail and thus may be considered in future research within this field of work. Issues that remain to be investigated in future include e.g.:

- Compare guidance related to lifting of countermeasures in different countries and-if existing!-check for applicability for long-lasting releases.

- Investigate effect of long-lasting emissions on monitoring strategies and capabilities.
- Conversion of release rates to measureable quantities (dose rates, air concentrations) to study preparedness related to monitoring.

- A long lasting low release rate of atmospheric discharge would probably require a very large capacity in airsampling monitoring.

- Identification of possible problems related to quantification of deposition during (residual) release.

- Study relative influence of peak releases and long lasting (residual) low release rate discharges to total impact and protection strategies.

- Check implementation of EU BSS (in different countries) on suitability for long-lasting releases.

- Is evacuation during passage of the plume nearly always preferable against sheltering (as stated in IAEA EPR 2013 document)?

- Check the influence of uncertainty (e.g. of wind prediction, model prognosis) on recommendations for protective actions and on decisions taken

- How to present uncertainties to decision makers?

Acknowledgement. The research leading to these results has received funding from the European Atomic Energy Community Seventh Framework Programme FP7/2012-2013 under grant agreement 323287.

\section{References}

Bujan A. et al. (2016) Emergency preparedness for long lasting releases - source terms, Radioprotection, 51 (HS2), S67-S71.

Montero M. et al. (2016) Emergency preparedness for long lasting releases - weather scenarios, Radioprotection, 51 (HS2), S73-S77.

Peltonen T. et al. (2016) Emergency preparedness for long lasting releases - assessment of radiological consequences, Radioprotection, 51 (HS2), S79-S81.

Cite this article as: F. Gering, B. Gerich, K. Arnold, T. Peltonen, T. Duranova, A. Bujan, J. Duran, L. Bohun, M. Montero, C. Trueba, L. Puijker, C. Twenhöfel, H. de Vries. Emergency preparedness for long lasting releases - overview and conclusions. Radioprotection 51 (HS2), S63-S65 (2016). 